\title{
X-ray diffraction reciprocal space mapping study of the thin film phase of pentacene
}

\author{
$\operatorname{AUTHOR}(S)$ :
}

Yoshida, H; Inaba, K; Sato, N

\section{CITATION:}

Yoshida, $H$...[et al]. X-ray diffraction reciprocal space mapping study of the thin film phase of pentacene. APPLIED PHYSICS LETTERS 2007, 90(18): 181930.

\section{ISSUE DATE:}

2007-04-30

URL:

http://hdl.handle.net/2433/50336

\section{RIGHT:}

Copyright 2007 American Institute of Physics. This article may be downloaded for personal use only. Any other use requires prior permission of the author and the American Institute of Physics. 


\title{
X-ray diffraction reciprocal space mapping study of the thin film phase of pentacene
}

\author{
Hiroyuki Yoshida ${ }^{a)}$ \\ Institute for Chemical Research, Kyoto University, Uji, Kyoto 611-0011, Japan \\ Katsuhiko Inaba \\ X-ray Research Laboratory, Rigaku Corporation, 3-9-12 Matsubara-cho, Akishima, Tokyo 196-8666, Japan \\ Naoki Sato \\ Institute for Chemical Research, Kyoto University, Uji, Kyoto 611-0011, Japan
}

(Received 15 February 2007; accepted 9 April 2007; published online 4 May 2007)

\begin{abstract}
The structure of the thin film phase of pentacene was investigated using $\mathrm{x}$-ray diffraction reciprocal space mapping (RSM). The crystal structure was found to be triclinic with the following lattice parameters: $a=0.593 \mathrm{~nm}, b=0.756 \mathrm{~nm}, c=1.565 \mathrm{~nm}, \alpha=98.6^{\circ}, \beta=93.3^{\circ}$, and $\gamma=89.8^{\circ}$. Atomic positions were determined by comparing the observed RSM diffraction intensities with theoretical calculations. (C) 2007 American Institute of Physics. [DOI: 10.1063/1.2736193]
\end{abstract}

Due to their relatively high hole mobility, pentacene thin films are promising candidates as working layer in organic field effect transistors (OFETs). ${ }^{1}$ In order to understand the transport properties, the electronic structure of pentacene thin films has been extensively investigated. For example, the intermolecular band dispersion was recently reported. ${ }^{2}$ As such electronic structure is strongly affected by the overlaps between the molecular orbitals of adjacent molecules, it is important to obtain precise information about the crystallographic structure of the film.

Pentacene has several polymorphs ${ }^{3-5}$ distinguished by the interlayer spacings along the $c^{*}$ axis. The structures of the "single crystal" $[d(001)=1.41 \mathrm{~nm}]$ (Refs. 6 and 7) and "bulk" $[d(001)=1.44 \mathrm{~nm}]$ (Ref. 8) phases have already been determined by $\mathrm{x}$-ray crystallography. On the other hand, thin films fabricated on insulating layers such as $\mathrm{SiO}_{2}$ often show a unique structure called the "thin film" phase that is characterized by an interlayer spacing of $d(001)=1.54 \mathrm{~nm}$. The structural information of this phase is particularly relevant because this polymorph is usually present in the working layers of pentacene OFETs. Complete structural data have not been obtained, however, since this phase is only found in films thinner than $100 \mathrm{~nm}$ and no appropriate method for structural analysis for such films existed.

The structure of the thin film phase has been investigated using electron diffraction (ED), ${ }^{5,9-12}$ grazing incidence $\mathrm{x}$-ray diffraction (GIXD) (also known as in-plane $\mathrm{x}$-ray diffraction), ${ }^{13-15}$ and the $\theta-2 \theta$ scan of x-ray diffraction. ${ }^{3-5,9}$ The former two methods only give the structural periodicity parallel to the substrate surface, while the $\theta-2 \theta$ scan gives only the vertical periodicity. If the $c^{*}$ axis is perpendicular to the substrate surface, only three parameters of the twodimensional lattice, that is, $a^{*}, b^{*}$, and $\gamma^{*}$ can be determined with GIXD and ED. The $c^{*}$ value is usually obtained from the $\theta-2 \theta$ scan.

A lattice in three dimensions (3D) is characterized by a set of six parameters $a, b, c, \alpha, \beta$, and $\gamma$ in real space, or equivalently, $a^{*}, b^{*}, c^{*}, \alpha^{*}, \beta^{*}$, and $\gamma^{*}$ in reciprocal space. With conventional methods, the $\alpha^{*}$ and $\beta^{*}$ values are not

${ }^{\text {a)} E l e c t r o n i c ~ m a i l: ~ y o s h i d a @ e . k u i c r . k y o t o-u . a c . j p ~}$ obtained. They are, however, indispensable in determining the structure of monoclinic and triclinic systems typical of organic crystals. As a result there is confusion regarding the crystal system of the thin film phase of pentacene: Both triclinic $^{5,10}$ and orthorhombic ${ }^{11}$ systems have been claimed.

In this work, we applied $\mathrm{x}$-ray reciprocal space mapping (RSM) to measure asymmetric as well as symmetric diffraction intensities in order to obtain a full set of lattice parameters for pentacene in the thin film phase. While this method is often used to examine expitaxially grown inorganic films, its application to the organic films is not straightforward. In most cases, the diffractions from the organic films are low order and low intensity due to large lattice size and poor crystallinity. We demonstrate, however, that by modifying the x-ray optics, it becomes possible to determine all of the six parameters in organic thin films including $\alpha^{*}$ and $\beta^{*}$. Furthermore, the atomic positions in a unit cell can be determined from the diffraction intensities. Such a detailed structure analysis, however, requires a large number of reflections with quantitatively reliable intensities. As this condition is not normally fulfilled in the thin film RSM measurement, we have compared the observed diffraction intensity pattern with those calculated on the basis of structural models.

A 50-nm-thick film of pentacene (purchased from Tokyo Kasei Kogyo Co., Ltd.) was grown by vacuum deposition on the naturally oxidized surface of a $\mathrm{Si}(100)$ wafer. The deposition rate was $0.1 \mathrm{~nm} \mathrm{~s}^{-1}$ and the pressure was lower than $1 \times 10^{-4} \mathrm{~Pa}$. All the $\mathrm{x}$-ray diffraction measurements were carried out in air on a Rigaku ATX-G diffractometer. ${ }^{16}$ The experimental setup is shown in Fig. 1. Cu $K \alpha$ x-ray radiation was monochromatized and collimated to a line shaped beam $\left(1 \times 10 \mathrm{~mm}^{2}\right)$ using a parabolic multilayer $\mathrm{x}$-ray mirror and a series of Soller slits. The diffracted $\mathrm{x}$ rays were collected with a crossed set of parallel slit analyzers and detected by a scintillation counter. The goniometer axis $\phi$ was aligned to the $c^{*}$ axis of the pentacene film which was confirmed to be normal to the substrate surface within the experimental uncertainty of $0.01^{\circ}$. There was no preferential orientation of the pentacene thin film around the $c^{*}$ axis.

Our experimental setup, shown in Fig. 1, is characterized by the additional detector motion along the $2 \theta \chi$ axis. RSM data are usually acquired with the two angles, $2 \theta$ and $\omega$, 


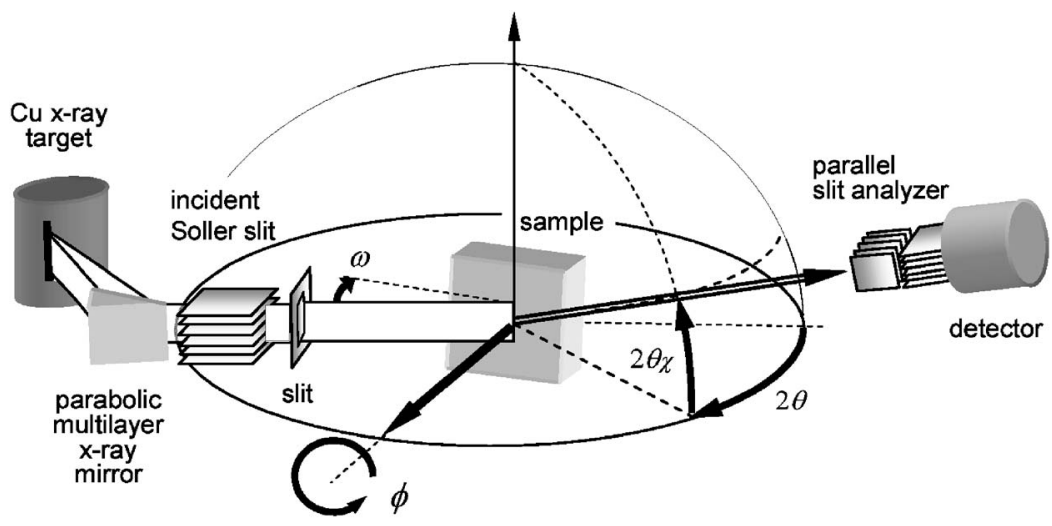

FIG. 1. Experimental setup for $x$-ray reciprocal space mapping optimized for measurements of organic thin films. Diffraction intensity data are collected over three angles $2 \theta, 2 \theta \chi$, and $\omega$.

scanned at a fixed angle $\phi$ (i.e., $2 \theta \chi=0$ in Fig. 1). Using different incident and detection angles, i.e., $2 \theta / 2 \neq \omega$, an asymmetric lattice plane is accessed that has a reciprocal lattice vector $\mathbf{Q}$ tilting by the angle $(2 \theta / 2-\omega)$ from the surface normal of the substrate. While this setup is simple and has high resolution, most of the asymmetric reflections with low $\mathbf{Q}$ cannot be examined due to the restriction imposed by $\omega>0$. This configuration is therefore inappropriate for the measurement of organic thin films which usually show only low angle diffractions. By scanning over the $2 \theta, \omega$, and $2 \theta \chi$ axes, we are able to obtain all the $\mathbf{Q}$ vectors that point above the sample surface. This setup also allows us to use the full beam size of the line-focused $\mathrm{x}$-ray beam by keeping both the incident $\mathrm{x}$-ray beam and the surface normal of the sample in the same plane, enabling high detection efficiency.

Figure 2 shows RSM patterns for $11 \ell, 02 \ell, 12 \ell$, and $20 \ell$ regions with $\ell=0$ and $\ell= \pm 1$. $c^{*}$ was independently evalu-

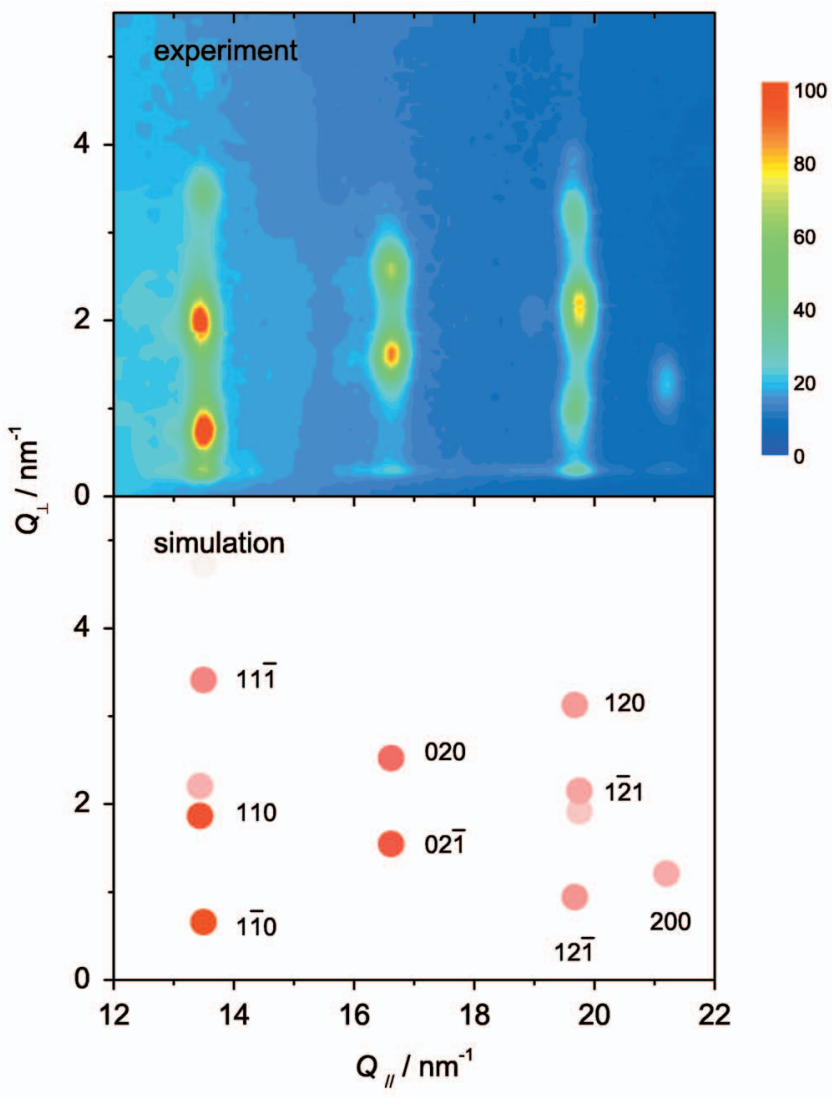

FIG. 2. (Color) Experimental (upper panel) and simulated (lower panel)

reciprocal space maps of the thin film phase of pentacene.

Downloaded 05 Mar 2008 to 130.54.110.22. Redistribution subject ated from the $\theta-2 \theta$ scan, while the other five lattice parameters were determined from RSM. Note that the spot corresponding to the $1 \overline{2} 1$ peak was observed at a slightly different $Q_{\|}$value compared to the 120 and $12 \overline{1}$ reflections, where $\gamma^{*}$ can be determined from the magnitude of the difference. In a previous GIXD study, ${ }^{14}$ this splitting of $h k \ell$ and $h \bar{k} \ell$ peaks was not observed, and the precise $\gamma^{*}$ value could not be determined. Our results show that the thin film phase of pentacene is triclinic with lattice parameters as follows: $a$ $=0.593 \mathrm{~nm}, b=0.756 \mathrm{~nm}, c=1.565 \mathrm{~nm}, \alpha=98.6^{\circ}, \beta=93.3^{\circ}$, $\gamma=89.8^{\circ}$. The cell volume is $V=0.693 \mathrm{~nm}^{3}$.

The lattice parameters along the substrate planes $a, b$, and $\gamma$ can be directly compared with the values obtained from ED, GIXD, and reflection high-energy electron diffraction experiments. They are in a good agreement with those of a pentacene monolayer on $\mathrm{SiO}_{2}$ (Ref. 13) as well as multilayered films on various substrates including $\mathrm{SiO}_{2},{ }^{14,17}$ self assembled monolayer (octadecyltrichlorosilane- and hexadimethylsilazane-) treated $\mathrm{SiO}_{2},{ }^{15}$ and $\mathrm{KCl},{ }^{12}$ suggesting that all these films together with the system examined in this work have the same crystal structure. On the other hand, the lattice parameters for films claimed to be of the bulk phase such as the epitaxially grown layers on $\mathrm{Bi}(001)$ (Ref. 18) and hydrogen-terminated $\mathrm{Si}(111){ }^{19}$ the organic solvent-treated multilayer, ${ }^{20}$ and the upperlayers in a heat-treated thick film on $\mathrm{SiO}_{2}$ (Refs. 17 and 20) are significantly different, indicating that these films have different polymorphs entirely.

3D lattice parameters have occasionally been derived from an ED pattern, in such cases where the $\alpha^{*}$ and $\beta^{*}$ values could be obtained directly from the ED patterns of tilted samples. The 3D lattice parameters for pentacene thin films on a $\mathrm{NaCl}$ substrate ${ }^{10}$ are close to ours within the limited accuracy of the ED method. On the other hand, when 3D values were estimated either by assuming orthorhombic symmetry $^{11}$ or by using a structural model, ${ }^{21}$ the values for the thin film phase on amorphous carbon do not agree with ours. These results demonstrate the advantage of our RSM measurement where accurate and reliable $\alpha^{*}$ and $\beta^{*}$ values can be measured.

The unit cell of the thin film phase should contain two molecules like the other polymorphs, ${ }^{6-8}$ since the cell volume is similar. As reflections of $h 00$ and $0 k 0$ with odd $h$ or $k$ values were not observed, ${ }^{14,20}$ it is clear that the two pentacene molecules in the unit cell are arranged in a herringbone manner; the projection of the structure along the $c$ axis (i.e., onto the $a^{*} b^{*}$ plane) belongs to the $p 2 g g$ space group. Using the lattice parameters together with this symmetry constraint, 
Thin film phase
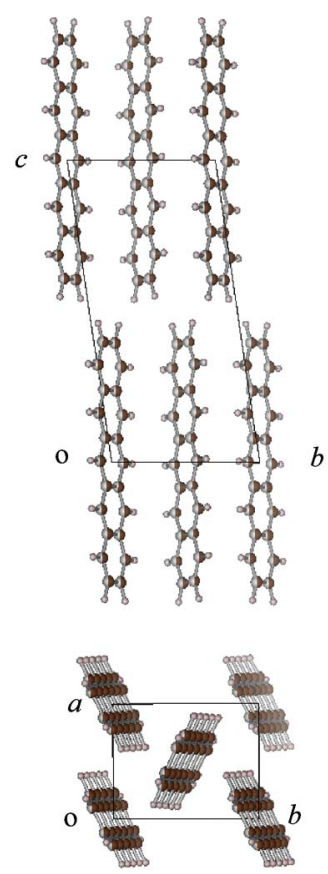

Bulk phase
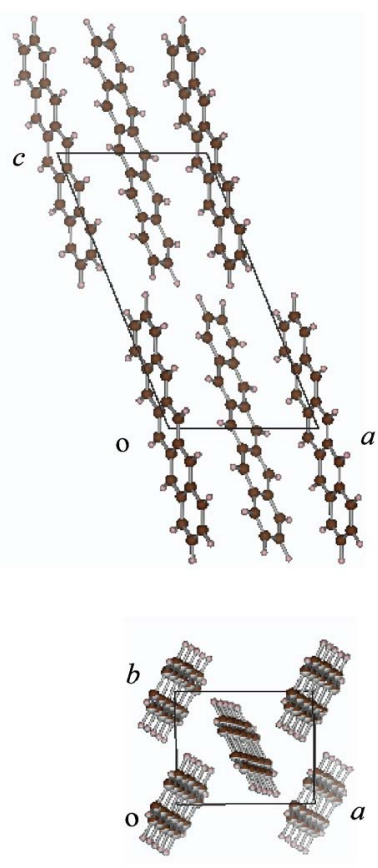

FIG. 3. (Color online) Structure of the thin film phase of pentacene determined from the $\mathrm{x}$-ray diffraction reciprocal space mapping and the atomatom potential calculation (left). The bulk phase structure from Ref. 8 is presented for comparison (right).

the lattice energies were calculated in order to find stable structures for the thin film phase.

The geometry of an isolated pentacene molecule was optimized with the B3LYP/6-31G $(d)$ method $^{22}$ and treated as a rigid body throughout the following calculations. Intermolecular interaction energies were calculated as a sum of atom-atom van der Waals interactions using the Dreiding force field. ${ }^{23}$ From the symmetry considerations mentioned above, only three degrees of freedom are left, i.e., those of the molecular orientation of one independent molecule in the unit cell. Lattice energies were calculated for randomly generated Euler angles of the molecular orientation. Four stable structures could be obtained without ambiguity as a result of the steep gradient in the intramolecular potentials induced by the fixed lattice parameters.

After further geometry optimization using the CERIUS $^{2}$ program (Accerlys) without the symmetry constraints, the diffraction patterns were calculated as reported previously. ${ }^{20}$ For the diffraction intensities, only the structure factors and the Lorenz and polarization corrections were included. Only one of the four simulated diffraction patterns matched the experimental RSM, as shown in Fig. 2. The corresponding structure, shown in Fig. 3 together with that of the bulk phase for comparison, was also energetically the most stable.

In this structure, the angle between the two molecular planes is $50.0^{\circ}$. This value nearly falls within the range of the interplane angles $\left(51^{\circ}-53^{\circ}\right)$ characterized by the herringbone structure for the other polymorphs of pentacene and other polyacene crystals. ${ }^{21}$ The molecular long axis is tilted from the surface normal by $5.7^{\circ}$ and $6.8^{\circ}$ for the two independent molecules. These angles are much smaller than the tilt angles of $25^{\circ}$ and $21^{\circ}$ reported for the single crystal ${ }^{6,7}$ and the bulk ${ }^{8}$ phases, respectively. They are also smaller than the value of $16^{\circ}-20^{\circ}$ estimated from the interlayer spacing and an evaluated molecular length. ${ }^{13,21}$

In conclusion, we have demonstrated that the x-ray RSM is a useful method to examine the structure of organic thin films that often have monoclinic or triclinic structures. Using RSM, the complete lattice parameters for the thin film phase of pentacene fabricated on $\mathrm{SiO}_{2}$ substrates were determined. The most probable structure was obtained by comparing the experimental diffraction intensities with those simulated for the structure calculated on the basis of the atom-atom potential method.

The authors thank T. Nemoto at Kyoto University for stimulating discussion on the pentacene film structures. The calculations were carried out at the Supercomputer Laboratory, Institute for Chemical Research, Kyoto University. This work was partly supported by a Grant-in-Aid for Scientific Research on Priority Areas of Molecular Conductors Grant No. 15073214 from the Ministry of Education, Culture, Sports, Science, and Technology of Japan.

${ }^{1}$ T. W. Kelley, P. F. Baude, C. Gerlach, D. E. Ender, D. Muyres, M. A. Haase, D. E. Vogel, and S. D. Theiss, Chem. Mater. 16, 4413 (2004).

${ }^{2}$ N. Koch, A. Vollmer, I. Salzmann, B. Nickel, H. Weiss, and J. P. Rabe, Phys. Rev. Lett. 96, 156803 (2006).

${ }^{3}$ C. D. Dimitrakopoulos, A. R. Brown, and A. Pomp, J. Appl. Phys. 80, 2501 (1996).

${ }^{4}$ I. P. M. Bouchoms, W. A. Schoonveld, J. Vrijmoeth, and T. M. Klapwijk, Synth. Met. 104, 175 (1999).

${ }^{5}$ C. C. Mattheus, A. B. Dros, J. Baas, G. T. Oostergetel, A. Meetsma, J. L. de Boer, and T. T. M. Palstra, Synth. Met. 138, 475 (2003).

${ }^{6}$ D. Holmes, S. Kumaraswamy, A. J. Matzger, and K. P. C. Vollhardt, Chem.-Eur. J. 5, 3399 (1999).

${ }^{7}$ C. C. Mattheus, A. B. Dros, J. Baas, A. Meetsma, J. L. de Boer, and T. T. M. Palstra, Acta Crystallogr., Sect. C: Cryst. Struct. Commun. 57, 939 (2001).

${ }^{8}$ R. B. Campbell, J. Trotter, and J. Monteath, Acta Crystallogr. 15, 289 (1962).

${ }^{9}$ T. Minakata, H. Imai, M. Ozaki, and K. Saco, J. Appl. Phys. 72, 5220 (1992).

${ }^{10}$ J. S. Wu and J. C. H. Spence, J. Appl. Crystallogr. 37, 78 (2004).

${ }^{11}$ L. F. Drummy and D. C. Martin, Adv. Mater. (Weinheim, Ger.) 17, 903 (2005).

${ }^{12}$ T. Kiyomura, T. Nemoto, T. Ogawa, T. Minari, K. Yoshida, H. Kurata, and S. Isoda, Jpn. J. Appl. Phys., Part 1 45, 401 (2006).

${ }^{13}$ S. E. Fritz, S. M. Martin, C. D. Frisbie, M. D. Ward, and M. F. Toney, J. Am. Chem. Soc. 126, 4084 (2004).

${ }^{14}$ R. Ruiz, A. C. Mayer, G. G. Malliaras, B. Nickel, G. Scoles, A. Kazimirov, H. Kim, R. L. Headrick, and Z. Islam, Appl. Phys. Lett. 85, 4926 (2004).

${ }^{15}$ H. C. Yang, T. J. Shin, M. M. Ling, K. Cho, C. Y. Ryu, and Z. N. Bao, J. Am. Chem. Soc. 127, 11542 (2005).

${ }^{16} \mathrm{~K}$. Omote and J. Harada, Adv. X-Ray Anal. 43, 192 (2000).

${ }^{17}$ T. Kakudate, N. Yoshimoto, and Y. Saito, Appl. Phys. Lett. 90, 081903 (2007).

${ }^{18}$ J. T. Sadowski, T. Nagao, S. Yaginuma, Y. Fujikawa, A. Al-Mahboob, K. Nakajima, T. Sakurai, G. E. Thayer, and R. M. Tromp, Appl. Phys. Lett. 86, 073109 (2005).

${ }^{19}$ T. Shimada, H. Nogawa, T. Hasegawa, R. Okada, H. Ichikawa, K. Ueno, and K. Saiki, Appl. Phys. Lett. 87, 061917 (2005).

${ }^{20}$ H. Yoshida and N. Sato, Appl. Phys. Lett. 89, 101919 (2006).

${ }^{21}$ C. C. Mattheus, G. A. de Wijs, R. A. de Groot, and T. T. M. Palstra, J. Am. Chem. Soc. 125, 6323 (2003).

${ }^{22}$ M. J. Frisch et al., Gaussian 03, Revision B. 03, Gaussian Inc., Pittsburgh, PA, 2003.

${ }^{23}$ S. L. Mayo, B. D. Olafson, and W. A. Goddard III, J. Phys. Chem. 94, 8897 (1990) 